\title{
Electrochemical skin conductance to detect sudomotor dysfunction, peripheral neuropathy and the risk of foot ulceration among Saudi patients with diabetes mellitus
}

Eman Sheshah, Amal Madanat*, Fahad Al-Greesheh, Dalal AL-Qaisi, Mohammad AL-Harbi, Reem Aman, Abdul Aziz AL-Ghamdi and Khaled AL-Madani

\begin{abstract}
Background: Sudomotor dysfunction is manifested clinically as abnormal sweating leading to dryness of feet skin and increased risk of foot ulceration. The aim of this study was to test the performance of foot electrochemical skin conductance (ESC) to detect diabetic peripheral neuropathy and the risk of foot ulceration against traditional methods in Saudi patients with diabetes mellitus.

Methods: This cross-sectional study was conducted on 296 Saudi patients with diabetes mellitus. Painful neuropathic symptoms were evaluated using the neuropathy symptom score (NSS). The risk of foot ulceration and diabetic peripheral neuropathy were determined using the neuropathy disability score (NDS). Vibration perception threshold (VPT) was assessed using neurothesiometer. Neurophysiological assessment of the right and left sural, peroneal and tibial nerves was performed in 222 participants. Diabetic peripheral neuropathy was defined according to the definition of the American Academy of Neurology. ESC was measured with Sudoscan.

Results: Feet-ESC decreased as the scores of sensory and motor function tests increased. Feet-ESC decreased as the NSS, NDS and severity of diabetic peripheral neuropathy increased. Sensitivity of feet-ESC $<50 \mu \mathrm{S}$ to detect diabetic peripheral neuropathy assessed by VPT $\geq 25 \mathrm{~V}, \mathrm{NDS} \geq 3, \mathrm{NDS} \geq 6$ was $90.1,61$ and $63.8 \%$ respectively and specificity 77,85 and $81.9 \%$ respectively. Sensitivity of feet-ESC $<70 \mu \mathrm{S}$ to detect diabetic peripheral neuropathy assessed by VPT $\geq 25$ V, NDS $\geq 3$, NDS $\geq 6$ was $100,80.6$ and $80.9 \%$ respectively. Sensitivity and specificity of feet-ESC $<70 \mu$ to detect confirmed-diabetic peripheral neuropathy were 67.5 and $58.9 \%$ respectively.
\end{abstract}

Conclusion: Sudoscan a simple and objective tool can be used to detect diabetic peripheral neuropathy and the risk of foot ulceration among patients with diabetes mellitus. Prospective studies to confirm our results are warranted.

Keywords: Sudomotor dysfunction, Sudoscan, Diabetic peripheral neuropathy, Neuropathy disability score

\footnotetext{
* Correspondence: madanatamal.y@gmail.com

Diabetes Care Center, King Salman Hospital (MOH), Ayesha Bint Abi Baker St.,

PO Box: 15169, Riyadh 11444, Kingdom of Saudi Arabia
}

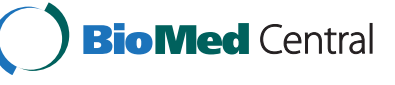

(c) 2016 The Author(s). Open Access This article is distributed under the terms of the Creative Commons Attribution 4.0 International License (http://creativecommons.org/licenses/by/4.0/), which permits unrestricted use, distribution, and reproduction in any medium, provided you give appropriate credit to the original author(s) and the source, provide a link to the Creative Commons license, and indicate if changes were made. The Creative Commons Public Domain Dedication waiver (http://creativecommons.org/publicdomain/zero/1.0/) applies to the data made available in this article, unless otherwise stated. 


\section{Background}

Diabetic autonomic neuropathy is a disorder of the autonomic nervous system affecting the cardiovascular, gastrointestinal and urogenital systems and sudomotor function in the setting of diabetes mellitus (DM) and metabolic derangements of pre-diabetes after exclusion of other causes [1].

In the context of diabetic peripheral neuropathy (DPN) sudomotor dysfunction (SMD) can occur in two phenotypes: either as one component of a generalized DPN, or concurrently in distal small fiber sensory polyneuropathy (SFSN) [2].

Sudomotor C-fibers are postganglionic, unmyelinated, cholinergic, sympathetic nerves that innervate sweat glands, and SMD is manifested clinically as abnormal sweating leading to skin dryness. Dryness of foot skin as a result of SMD is associated increased risk of foot ulceration (FU) [3].

Prevention of FU in patients with DM continues to represent an important issue, as the steady rise in the prevalence of DM is leading to a persistent burden of DM-related complications including lower limb amputations [4]. Approximately $84 \%$ of non-traumatic amputations occurring in diabetes are preceded by FU [5]. While peripheral artery disease accounts for an increased risk of FU in only $35 \%$ of cases, DPN contributes to $78 \%$ of the risk of FU, and together with foot deformity and repetitive trauma forms the clinical triad that leads to FU $[5,6]$.

As such, every effort is needed to detect DPN including SMD early in the course of the disease in order to identify those patients in need of special care to minimize the risk of FU and prevent limb loss. Utilization of tests to assess SMD in daily practice has been limited, as the known standardized methods to do so are either invasive, complex, time-consuming or require specialized equipment and training [2].

Recent introduction of sudorimetry technology using Sudoscan which measures electrochemical skin conductance (ESC) of the hands and feet based on reverse iontophoresis and chronoamperometry has allowed rapid, noninvasive, robust, accurate assessment of sudomotor and small nerve fiber function [7]. ESC measurement requires little technical training and no calculations and can easily be integrated into daily practice.

Over the past three decades the prevalence of DM in Saudi Arabia has increased approximately 10-fold [8]. As DPN is the earliest and most common long term complication of DM $[5,9]$, it is imperative to validate and utilize novel screening and diagnostic methods [10], in particular those that are simple, non-invasive, easy to use at a point of care and that have the potential to overcome barriers to screening for and detecting DPN including SMD.
The aim of our study was to test the performance of feet-ESC to detect DPN and the risk of FU against traditional methods among Saudi patients with DM.

\section{Methods}

A cross-sectional observational study was conducted at the diabetes care center, King Salman hospital in Riyadh, Kingdom of Saudi Arabia between January and May 2012. Informed consent was obtained from all participants.

\section{Study population}

Participants in the study consisted of two hundred ninety six Saudi Arabian patients with DM referred for the first time from primary care health centers for general diabetes care. Exclusion criteria included patients younger than 18or older than 65 years of age; patients with secondary causes of DPN, peripheral vascular disease or active foot ulcer; or patients taking drugs that affect autonomic function testing such as $\beta$-blockers or atropine.

\section{Clinical assessment}

Physical examination including detailed feet assessment was conducted by physicians as described by Boulton AJ et al [11]. Characteristics of the appearance of the feet were documented for the presence of dryness, fissures, calluses and deformities.

Painful neuropathic symptoms (PNS) were evaluated using the neuropathy symptom score (NSS) [12]. Symptoms were considered positive if NSS $\geq 5$. Diabetic peripheral neuropathy and the risk of FU were determined using the neuropathy disability score (NDS). DPN was present if $\mathrm{NDS} \geq 3$. Risk of FU was present if $\mathrm{NDS} \geq 6$ [12]. Severity of DPN was graded according to NDS scores: None (0-2), Mild (3-5), Moderate (6-8), Severe (9-10) [12]. Scores for NDS were derived from ankle reflex testing using a reflex hammer, vibration sensation using a $128 \mathrm{~Hz}$ tuning fork, pain sensitivity using the neurotip and differences in temperature sensation using warm cold rod. Pressure sensation was tested using a 10-g monofilament as described by Boulton AJ et al [11]. Quantitative sensory testing (QST) to determine vibration perception threshold (VPT) was performed using a neurothesiometer on both halluces. Complete blood count was performed using (Hematology Analyzer, Sysmex XT 2000i), HbA1c was performed using (Dimension Xpand ${ }^{\mathrm{TM}}$ Rxl max, Hemoglobin A1c), the method has been certified for precision and accuracy by the National Glycohemoglobin Standardization Program (NGSP) ensuring clinical results consistent with the findings of the Diabetes Control and Complication Trial (DCCT). Blood chemistry was performed in the morning after $12 \mathrm{~h}$ fasting using

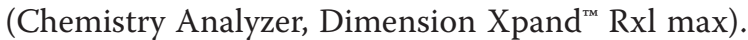




\section{Neurophysiological assessment}

Nerve conduction study was performed in 222 participants using Nicolet Viking Quest, VIASYS Healthcare Inc Neurocare Group USA. Right and left sural sensory amplitude and conduction velocity and peroneal and tibial motor amplitudes, latencies and conduction velocities were measured. Results were compared to age specific normal values to determine nerve conduction study abnormality. Participants were then classified as having normal, subclinical (ie nerve conduction abnormality but without symptoms or signs of DPN) or confirmed DPN as described by Tesfaye S et al 2010 [1]. Confirmed DPN was present if the participant had at least one symptom or sign of DPN and one or more abnormal nerve conduction test in both sural (sensory) and peroneal or tibial (motor) nerves in accordance with the case definition of DPN described by the American Academy of Neurology [13].

\section{Sudomotor assessment}

Measurement of ESC of both hands and feet was performed using Sudoscan. Participants placed their hands and feet on two sets of large-stainless-steel electrodes which were connected to a computer for recording and data management. Electrochemical skin conductance is the ratio of the current measured over the constant power applied expressed in micro-Simens $(\mu S)$ for the hands and feet (right and left sides). Through reverse iontophoresis, the device generates voltage to the cathode and a current (intensity of around $0.2 \mathrm{~mA}$ ) occur between the anode and cathode proportional to sweat chloride concentration. At low voltage $(<10 \mathrm{~V})$ the stratum corneum is electrically insulating and only sweat gland ducts are conductive. Sudomotor dysfunction is absent if measured hands-ESC is $\geq 60 \mu \mathrm{S}$ or feet$\mathrm{ESC} \geq 70 \mu \mathrm{S}$. Moderate $\mathrm{SMD}$ is present if measured hands-ESC is $\geq 40$ but $<60 \mu \mathrm{S}$ or feet-ESC is $\geq 50$ but $<$ $70 \mu \mathrm{S}$. Severe SMD is present if measured hands-ESC is $<40 \mu \mathrm{S}$ or feet-ESC is $<50 \mu \mathrm{S}$. These thresholds have been defined based on previous studies [14-16]. The test lasts less than $3 \mathrm{~min}$ and is painless.

\section{Statistical analysis}

Data are presented as mean \pm SD or percentages. Pearson's correlation coefficient was used to evaluate the relationship between feet-ESC and clinical and biochemical variables as well as bedside-tests, QST, and neurophysiologic testing to detect DPN. Variances between variables were calculated using an independent $T$ test. For all the tests a $P$ value of 0.05 or less was used for statistical significance. Sensitivity and specificity for feet-ESC to detect DPN and the risk of FU were calculated against the cutoff values of $\mathrm{VPT} \geq 25 \mathrm{~V}, \mathrm{NDS} \geq 3$, confirmed-DPN for the presence of DPN and NDS $\geq 6$ for the presence of increased risk of FU using Bland-Altman plots [12, 17, 18]. Receiver operating characteristic (ROC) curves were constructed and area under the curve (AUC) calculated. Statistical Package for Social Sciences (SPSS) version 20 for windows was used for statistical analysis.

\section{Results}

The mean age of the participants was $46.7 \pm 11.2$ years. Type 2 DM (T2DM) was present in $91.9 \%$ of the participants and $8.1 \%$ had type 1 DM. Male to female ratio was 1.01. Duration of DM was $6.97 \pm 7.1$ years. Body mass index was $30.9 \pm 7.1 \mathrm{~kg} / \mathrm{m}^{2}$. History of $\mathrm{FU}$ was present in (11) $3.7 \%$ of the participants. Hypertension and dyslipidemia were present in (109) $36.8 \%$ and (144) $48.6 \%$ of the participants respectively and $80.1 \%$ led a sedentary life-style. Diabetic retinopathy was present in (16) $5.4 \%$ of the participants.

Painful neuropathic symptoms (PNS) were present in (154) $52 \%$ of the participants and moderate PNS as defined by NSS $\geq 5$ were present in 53 (18.2\%). DPN as defined by NDS $\geq 3$ was present in (67) $22.6 \%$ of the participants and $15.9 \%$ had increased risk of FU as defined by NDS $\geq 6$. DPN of moderate severity was present in $35(11.8 \%)$ of the participants.

Quantitative sudorimetry using hands and feet-ESC was performed in all patients. As hands-ESC correlated significantly with feet-ESC (Table 1), we used only feetESC to analyze the relationship of sudorimetry with the other variables. Among all participants, 137 (46.3\%) had no SMD: feet-ESC $\geq 70 \mu \mathrm{S}, 84(28.4 \%)$ had moderate SMD: feet-ESC $<70-\geq 50 \mu \mathrm{S}$ and 75 (25.3\%) had severe SMD: feet-ESC $<50 \mu \mathrm{S}$.

There was a significant negative correlation between feet-ESC and age, duration of DM and a past history of FU (-0.234 $p<0.0001),(-0.301 p<0$.003), and $(-0.366$ $p<0.0001)$ respectively. Feet-ESC decreased in the presence of diabetic retinopathy $(-0.170 p<0$.003) and elevation of systolic blood pressure $(-0.129, p<0$.036) Feet-ESC also decreased with rising levels of serum creatinine $(-0.143, p<0.014)$ and uric acid $(-0.162, p<0$ .008). Notably feet-ESC increased with increasing levels of physical activity $(0.171, p<0.0001)$. Analysis of the relationship between feet-ESC and the levels of fasting blood glucose (FBG) and HbA1c showed an insignificant inverse relationship: $(-0.026, p<0.66)$, and $(-0.062, p<$ $0.31)$ respectively. However there was a positive correlation between both FBG and HbA1c and patients who are in the category of severe SMD (feet-ESC $<50 \mu \mathrm{S}$ ) as a group, $(0.118, p<0.044),(0.134, p<0.029)$ respectively.

Table 1 presents trends in the correlation coefficients between feet-ESC and tests of peripheral nerve function. The values of feet-ESC decreased as the bedside sensory and motor function tests and VPT assessed quantitatively by neurothesiometer increased. The values of feet- 
Table 1 Pearson's correlation coefficient of feet-ESC with tests of peripheral nerve function among the participants

\begin{tabular}{llll}
\hline No & Tests & CC & $P$ \\
\hline 1 & ESC-hands & 0.592 & $<0.0001$ \\
2 & Pain sensitivity & 0.406 & $<0.0001$ \\
3 & Differences in temperature perception & 0.304 & $<0.0001$ \\
4 & Achilles Reflex & -0.405 & $<0.0001$ \\
5 & Vibration perception by $128 \mathrm{~Hz}$ tuning fork & -0.391 & $<0.0001$ \\
7 & Pressure perception by $10 \mathrm{~g}-\mathrm{MF}$ & -0.300 & $<0.0001$ \\
8 & QST by neurothesiometer:VPT $\geq 25 \mathrm{~V}$ & -0.383 & $<0.0001$ \\
9 & Sural nerve amplitude & 0.163 & $<0.014$ \\
10 & Sural nerve velocity & 0.249 & $<0.0001$ \\
11 & Peroneal nerve amplitude & 0.184 & $<0.006$ \\
12 & Peroneal nerve velocity & 0.278 & $<0.0001$ \\
13 & Painful DPN by NSS $\geq 5$ & -0.230 & $<0.0001$ \\
14 & DPN by NDS $\geq 3$ & -0.469 & $<0.0001$ \\
15 & Risk of foot ulcer by NDS $\geq 6$ & -0.398 & $<0.0001$ \\
16 & Severity of DPN by NDS $\geq 3$ & -0.442 & $<0.0001$ \\
\hline
\end{tabular}

DPN diabetic peripheral neuropathy. ESC electrochemical skin conductance. NSS Neuropathy symptom score. NDS neuropathy disability score. QST quantitative sensory testing. VPT vibration perception threshold. 10-g MF 10 gram monofilament. CC correlation coefficient

ESC also decreased with increasing scores of NSS, NDS $\geq 3$, and NDS $\geq 6$ reflecting the presence of PNS, DPN and the risk of foot ulceration (RFU). As the severity of DPN increased, the values of feet-ESC decreased. ESC-feet decreased as the amplitude and conduction velocity of the sensory and motor nerves decreased. Notably there was a positive correlation between feet-ESC and both pain sensation and differences in temperature sensation.

Table 2 presents variances in the mean feet-ESC between participants with and without PNS, DPN, increased risk of FU and confirmed DPN. There was a significant decrease in feet-ESC among participants with DPN compared with those without DPN. Further analysis showed significantly lower values of feet-ESC in patients with confirmed DPN as compared with participants with subclinical DPN $(54.7 \pm 24.8$ vs $70.2 \pm$ 14.14 $\mu$ S; $P<0.0001)$.

Table 2 Variances in feet-ESC between participants without or with painful neuropathic symptoms, diabetic peripheral neuropathy, RFU, and confirmed neuropathy

\begin{tabular}{lllll}
\hline & NSS $\geq 5$ & NDS $\geq 3$ & NDS $\geq 6$ & Confirmed-DPN \\
\hline No & $67.1 \pm 18.4 \mu S$ & $67.4 \pm 16.9 \mu S$ & $65.6 \pm 18.8 \mu S$ & $67.6 \pm 16.9 \mu S$ \\
Yes & $57.1 \pm 23.7 \mu S$ & $42.9 \pm 26.1 \mu S$ & $41.9 \pm 25.9 \mu S$ & $53.7 \pm 24.7 \mu S$ \\
$P$ & $<0.0001$ & $<0.0001$ & $<0.0001$ & $<0.0001$ \\
\hline
\end{tabular}

$D P N$ diabetic peripheral neuropathy, NSS Neuropathy symptom score. NDS neuropathy disability score. RFU risk of foot ulceration
Table 3 demonstrates the performance of severe SMD

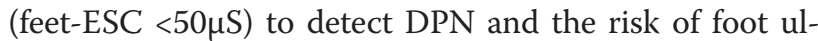
ceration, against the traditional tests, in particular: $\mathrm{VPT} \geq 25 \mathrm{~V}, \mathrm{NDS} \geq 3$ and NDS $\geq 6$. Feet-ESC $<50 \mu \mathrm{S}$ was sensitive and specific in detecting DPN and the risk of FU among participants. Similarly, feet-ECS $<70 \mu S$ was highly sensitive but less specific in detecting DPN assessed by VPT, NDS and confirmed DPN (defined by the criteria of the American Academy of Neurology 2005 [13]) with a notable high positive predictive (PPV) value for confirmed DPN. Figure 1 demonstrates the ROC curve of feet-ESC to reflect DPN assessed by $\mathrm{VPT} \geq 25 \mathrm{~V}$.

\section{Discussion}

We demonstrated that in Saudi patients with DM, severe SMD as defined by a feet-ESC threshold below $50 \mu \mathrm{S}$ was sensitive and highly specific in detecting DPN assessed by VPT and NDS. Feet-ESC $<50 \mu \mathrm{S}$ was sensitive and specific in detecting the risk of $\mathrm{FU}$ as well.

Furthermore feet-ESC threshold below $70 \mu \mathrm{S}$, showed moderate sensitivity to detect confirmed-DPN assessed by neurophysiological studies and classified according to the American Academy of Neurology 2005 criteria. In an earlier publication Casellini $\mathrm{C}$ et al showed that feetESC exhibited high sensitivity and specificity to detect DPN evaluated by the Neuropathy Impairment ScoreLower Legs (NIS-LL) [14]. In patients with type $1 \mathrm{DM}$, Selvarajah D et al have demonstrated that feet-ESC was sensitive and specific to identify confirmed-DPN classified according to AAN criteria [19].

Additionally we showed that feet-ESC was able to differentiate between participants with and without DPN assessed by NDS and neurophysiological testing. Our

Table 3 Performance of feet-ESC to detect diabetic peripheral neuropathy and the RFU against traditional tests

\begin{tabular}{|c|c|c|c|c|c|c|c|}
\hline & \multicolumn{7}{|c|}{ Feet-ESC $<50 \mu \mathrm{S}$} \\
\hline & Sensitivity & Specificity & $A \cup C$ & PPV & NPV & $+\mathrm{LH}$ & $-\mathrm{LH}$ \\
\hline DPN:VPT $\geq 25 \vee$ & 90.1 & 77 & 0.841 & 13 & 99 & 3.98 & 0.12 \\
\hline DPN:NDS $\geq 3$ & 61.2 & 85.2 & 0.732 & 54 & 85 & 4.12 & 0.46 \\
\hline RFU:NDS $\geq 6$ & 63.8 & 81.9 & 0.729 & 40 & 92 & 3.53 & 0.44 \\
\hline \multirow[t]{2}{*}{ Confirmed-DPN } & 38.4 & 91 & 0.648 & 85.7 & 52 & 4.3 & 0.67 \\
\hline & \multicolumn{7}{|c|}{ Feet-ESC $<70 \mu S$} \\
\hline DPN:VPT $\geq 25 \vee$ & 100 & 46 & 0.732 & 6.7 & 100 & 1.86 & 0 \\
\hline DPN:NDS $\geq 3$ & 80.6 & 51 & 0.663 & 32.5 & 90 & 1.66 & 0.38 \\
\hline RFU:NDS $\geq 6$ & 80.9 & 49 & 0.651 & 23 & 93 & 1.59 & 0.39 \\
\hline Confirmed-DPN & 67.2 & 58.9 & 0.63 & 93 & 56 & 1.6 & 0.56 \\
\hline
\end{tabular}

$D P N$ diabetic peripheral neuropathy. RFU risk of foot ulceration. ESC electrochemical skin conductance. VPT vibration perception threshold. NDS neuropathy disability score. AUC area under the curve. PPV positive predictive value. NPV negative predictive value. $+L H$ positive likelihood. $-L H$ negative likelihood 


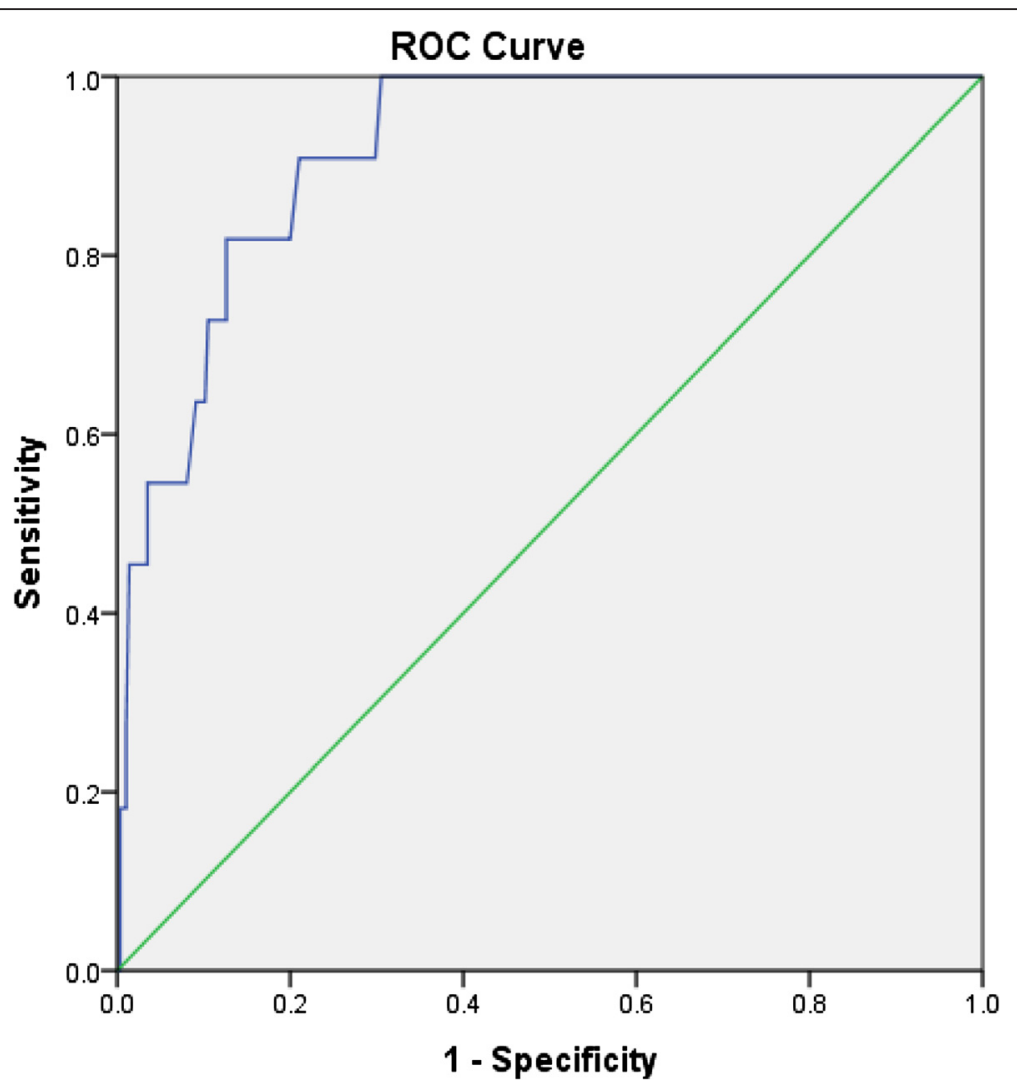

Fig. 1 Feet-ESC receiver-operating characteristic curve ROC to reflect diabetic peripheral neuropathy assessed by VPT $\geq 25 \mathrm{~V}$. Area under the curve $=0.918, P<0.0001$

results are in agreement with results reported in other studies. [14, 19, 20] These observations indicate that sudorimetry using feet-ESC relates significantly to tests of sensory and motor nerve function that are used to detect large fiber-sensory polyneuropathy (LFSN).

On the other hand we have shown that $53.7 \%$ of the participants had either moderate or severe SMD assessed by feet-ESC. Feet-ESC positively correlated with tests to detect pain sensitivity and differences in temperature sensation, both of which are bedside tests, used to assess small fiber nerve function. Although assessment of symptoms and even quantitative sensory tests for cold, warm and pain sensitivity lack precision in detecting small fiber nerve function [6] Smith et al have shown similar performance of feet-ESC compared to intraepidermal nerve fiber density (IENFD) obtained from skin biopsy that is considered the gold standard for diagnosing small fiber sensory neuropathy (SFSN) [15].

Together these results do not imply that ESC identifies all forms of DPN, but rather reflect its ability to detect the involvement of small $\mathrm{C}$-fiber nerve dysfunction in a generalized DPN in addition to the concurrent occurrence of autonomic and somatic C-fiber dysfunction in SFSN. This fact confers a potential role for ESC to screen for both LFSN and SFSN in addition to SMD. Being simple, non-invasive, quick, and objective and requiring no training or special patient preparation qualifies ESC as a suitable measurement tool to screen for DPN in busy diabetes clinics.

The strength of our study lies in the fact that we performed neurophysiological tests on a large number of participants (222) to define DPN in addition to bedside tests.

We also demonstrated that in Saudi patients with DM, quantitative sudorimetry using feet-ESC was related not only to traditional tests for identifying DPN but also to the factors that are associated with increased risk for developing DPN. Feet-ESC decreased with increasing age, duration of DM, systolic blood pressure and a past history of FU. Earlier Yajnik et al demonstrated a positive correlation of feet-ESC with age and duration of DM [20]. A decrease in the feet-ESC levels was observed with rising levels of serum uric acid. This is in agreement with results reported by Papanas $\mathrm{N}$ et al who demonstrated that increased levels of uric acid represents a risk factor for DPN in general and SMD in particular [21, 22].

Analysis of the relationship between indices of glycemic control and feet-ESC showed a significant positive correlation between both fasting blood glucose 
levels, $\mathrm{HbA1c}$ and feet-ESC threshold of severe SMD (feet-ESC $<50 \mu \mathrm{S}$ ) that probably represents established SMD. Results are in agreement with findings by Yajnik et al, [20] and imply that uncontrolled DM increases the risk of developing SMD. Decreased feet-ESC was also associated with the presence of diabetic retinopathy and increasing levels of serum creatinine. Gin et al also demonstrated an inverse relationship between ESC and diabetic retinopathy $(-0.42, p<0.0001)$ [23]. The relationship demonstrated in our study between indices of blood glucose control and severe SMD ie (feet-ESC $<50$ $\mu S)$, the presence of diabetic retinopathy and increasing levels of creatinine (the three of which represent indicators of diabetic microvascular complications) is in agreement with the results of the landmark studies that have shown a direct effect of glycemic control on the development of diabetic microvascular complications including DPN that encompasses SMD as well [24, 25]. It is worth mentioning that several recent studies have suggested utilization of a risk score based on ESC as a tool to screen for chronic kidney disease and microvascular complications [16, 26, 27].

An important finding of the study is the relationship between feet-ESC and the level of physical activity among the participants. Participants with a sedentary life-style were at increased risk of SMD reflected by decreased feetESC. This result emphasizes the role of PA in protecting sudomotor small C-fiber nerves and is in agreement with results reported by Raisanen A et al who demonstrated a significant increase in estimated $\mathrm{VO} 2 \mathrm{max}$ and hands and feet-ESC observed after lifestyle intervention [28].

We did not compare sudorimetry using ESC with other traditional tests to detect autonomic dysfunction. This might be considered a weakness of our study; however others have shown that ESC significantly correlated with the quantitative autonomic testing and with the quantitative sudomotor axon reflex testing $[14,15]$.

Our study has demonstrated that more than half of the studied Saudi patients with DM newly referred to a specialized diabetes center had SMD assessed quantitatively using ESC and could thus be at risk of developing FU. In this regard it is worth mentioning that a recent cohort study has demonstrated that the prevalence of diabetic foot complications among Saudi Arabian patients with DM is $3.3 \%$, and is within the estimated international range [29]. This burden is expected to grow as the prevalence of DM continues to rise in Saudi Arabia [8]. Simplicity and reliability are therefore prerequisites for a screening method to identify patients at risk of $\mathrm{FU}$, to be widely utilized as the prevalence of $\mathrm{DM}$ continues to rise. The study showed that measuring ESC is a simple, noninvasive, reliable, quantitative test that can be introduced at a point of care to screen for SMD, DPN and the risk of FU. Utilization of ESC will simplify screening for DPN to identify patients at risk who are in need of special care to prevent limb loss. Further studies are warranted to support the results and prospective studies should explore the potential of ESC to predict the development of FU and test the effectiveness of interventions to prevent SMD and FU.

\section{Conclusion}

Our study demonstrated that measuring feet-ESC is a simple, noninvasive method with sufficient sensitivity and specificity to identify patients with DM who are at risk of FU.

\section{Abbreviations \\ DM, diabetes mellitus; DPN, diabetic peripheral neuropathy; ESC, electrochemical skin conductance; FU, foot ulceration; NDS, neuropathy disability score; NSS, neuropathy symptom score; PNS, painful neuropathic symptoms; QST, quantitative sensory testing; SMD, sudomotor dysfunction; VPT, vibration perception threshold}

\section{Acknowledgments}

The authors wish to express gratitude to Nurses in the Neuro-Lab: Eman AL-Zahrani and Reem AL-Esmary. Part of the study was presented as an oral presentation during the First Clinical Congress \& Gulf Chapter of the American Association of Clinical Endocrinologists meeting in Abu Dhabi $3^{\text {rd }}$ October 2013

\section{Funding}

None.

\section{Availability of data and materials}

All data that support the conclusions of the article are available as SPSS program upon request.

\section{Authors' contributions}

ES participated in the design of the study, researched data and draft review. AM contributed to the concept and design of the study, researched data, performed the statistical analysis and drafted the manuscript. FG participated in supervising neurophysiological testing and draft review. DQ participated in data acquisition and draft review. MH participated in draft review. RA participated in data acquisition and draft review. AG participated in draft review. KM participated in data acquisition and draft review. The manuscript has been read and approved by all authors.

\section{Competing interests}

The authors declare that they have no competing interests.

\section{Consent for publication}

Not applicable.

\section{Ethics approval and consent to participate}

The study protocol was approved by the Ethics Committee of the King Salman Hospital, Ministry of Health, Riyadh, Kingdom of Saudi Arabia. Informed consent was obtained from all participants.

Received: 21 May 2016 Accepted: 2 August 2016

Published online: 05 August 2016

\section{References}

1. Tesfaye S, Boulton AJ, Dyck PJ, Freeman R, Horowitz M, Kempler P, et al. Diabetic Neuropathies: Update on definitions, diagnostic criteria, estimation of severity, and treatments. Diabetes Care. 2010;33:2285-93.

2. England JD, Gronseth GS, Franklin G. Practice parameter: Evaluation of distal symmetric polyneuropathy: Role of autonomic testing, nerve biopsy, and skin biopsy (an evidence-based review): Report of the American Academy of Neurology, American Association of Neuromuscular and Electrodiagnostic Medicine, and American Academy of Physical Medicine and Rehabilitation. Neurology. 2009;72:177-84. 
3. Tentolouris N, Marinou K, Kokotis P, Karanti A, Diakoumopoulou E, Katsilambros N. Sudomotor dysfunction is associated with foot ulceration in diabetes. Diabet Med. 2009;26:302-5.

4. Gregg EW, Li Y, Wang J, Burrows NR, Ali MK, Rolka D, et al. Changes in diabetes-related complications in the United States, 1990-2010. N Engl J Med. 2014;370:1514-23.

5. Barshes NR, Sigireddi M, Wrobel JS, Mahankali A, Robbins JM, Kouqias P, et al. The system of care for diabetic foot: objectives, outcomes, opportunities. Diabet Foot Ankle. 2013;4:10

6. Boulton AJ. What you can't feel can hurt you. J Vasc Surg. 2010;52:28S-30S.

7. Vinik Al, Nevoret ML, Casellini C. The new age of sudomotor function testing: a sensitive and specific biomarker for diagnosis, estimation of severity, monitoring progression and regression in response to intervention. Front Endocrinol. 2015;6:94

8. AL Dawish MA, Robert AA, Braham R, AL Hayek AA, AL Saeed A, Ahmed RA, et al. Diabetes mellitus in Saudi Arabia: a review of the recent literature. Curr Diabetes Rev 2015 July 2015 [Epub ahead of print].

9. Vinik Al, Mitchell BD, Leichter SB, Wagner AL, O'Brian JT, Georges LP. Epidemiology of the complications of diabetes. In: Leslie RDG, Robbins DC editors. Diabetes: Clinical Science in Practice. Cambridge, United Kingdom: Cambridge University Press; 1994. p. 221-87.

10. Papanas N, Ziegler D. New vistas in the diagnosis of diabetic polyneuropathy. Endocrine. 2014:47:690-8.

11. Boulton AJ, Armstrong DG, Albert SF, Frykberg RJ, Hellman R, Kirkman MS, et al. Comprehensive foot examination and risk assessment: a report of the Task Force of the Foot care Interest Group of the American Diabetes Association, with endorsement by the American Association of Clinical Endocrinologists. Dibetes Care. 2008;31:1679-85.

12. Abbot CA, Carrington AL, Ashe H, Bath S, Every LS, Griffths J, et al. The North-West Diabetes Foot Care Study: incidence of, and risk factors for, new diabetic foot ulceration in a community based patient cohort. Diabet Med. 2002:10:377-84

13. England JD, Gronseth GS, Franklin G, Miller RG, Asbury AK, Carter GT, et al. Distal Symmetrical Polyneuropathy: Definition for Clinical Research: report of the American Academy of Neurology, the American Association of Electrodiagnostic Medicine and the American Academy of Physical Medicine and Rehabilitation. Neurology. 2005;64:199-207.

14. Casellini CM, Parson HK, Richardson MS, Nevoret ML, Vinik Al. Sudoscan, a noninvasive tool for detecting diabetic small fiber neuropathy and autonomic dysfunction. Diabetes Technol Ther. 2013;15:948-53.

15. Smith AG, Lessard M, Reyna S, Doudova M, Singleton JR. The diagnostic utility of Sudoscan for distal symmetric peripheral neuropathy. J Diabetes Complications. 2014;28:511-6.

16. Freedman BI, Bowden DW, Smith SC, Xu J, Divers J. Relationships between electrochemical skin conductance and kidney disease in type 2 diabetes. J Diabetes Complications. 2014;28:56-60.

17. Young MJ, Breddy JL, Veves A, Boulton AJM. The prediction of diabetic neuropathic foot ulceration using vibration perceptions. A prospective study. Diabetes Care. 1994;17:557-61.

18. Altman DG, Bland JM. Diagnostic tests 1: sensitivity and specificity. Br Med J. 1994;308:308-52.

19. Selvarajah D, Cash T, Davies J, Sankar A, Rao G, Grieg M, et al. Sudoscan: A simple, rapid, and objective method with potential for screening for diabetic peripheral neuropathy. PLoS One. 2015;10:e0138224.

20. Yajnik CS, Kantikar W, Pande AJ, Deslypere JP. Quick and simple evaluation of sudomotor function for screening of diabetic neuropathy. ISRN Endocrinol. 2012;2012:103714.

21. Papanas N, Katsiki N, Papatheodorou K, Demetriou M, Papazolgou D, Gioka $T$, et al. Peripheral neuropathy is associated with increased serum levels of uric acid in type 2 diabetes mellitus. Angiology. 2011;62:291-5.

22. Papanas N, Demetriou M, Katsiki N, Papatheodorou K, Papazoglou D, Gioka T, et al. Increased Serum Levels of Uric Acid Are Associated with Sudomotor Dysfunction in Subjects with Type 2 DiabetesMellitus. Exp Diab Res. 2011; 2011:346051.

23. Gin H, Baudoin R, Raffaitin CH, Rigalleau V, Gonzalez C. Non-invasive and quantitative assessment of sudomotor function for peripheral diabetic neuropathy evaluation. Diabetes Metab. 2011;37:527-32.

24. The Diabetes Control and Complications Trial Research Group. The effect of intensive treatment of diabetes on the development and progression of long-term complications in insulin-dependent diabetes mellitus. N Engl J Med. 1993;329:977-86.
25. UK Prospective Diabetes Study (UKPDS) Group.Intensive blood-glucose control with sulphonylureas or insulin compared with conventional treatment and risk of complications in patients with type 2 diabetes (UKPDS 33). Lancet. 1998;352(9131):837-53.

26. Luk AO, Fu W, Li X, Ozaki R, Chung HY, Wong RY, et al. The Clinical Utility of SUDOSCAN in Chronic Kidney Disease in Chinese Patients with Type 2 Diabetes. PLoS One. 2015;10:e0134981.

27. Eranki VG, Santosh R, Rajitha K, Pillai A, Sowmya P, Dupin J, et al. Sudomotor function assessment as a screening tool for microvascular complications in type 2 diabetes. Diabetes Res Clin Pract. 2013;101:e11-3.

28. Raisanen A, Eklund J, Calvet J-H, Tuomilehto J. Sudomotor function as a tool for cardiorespiratory fitness level evaluation: comparison withVO2max. Int J Environ Res Public Health. 2014;11:5839-48.

29. AL-Rubeaan K, AL-Derwish M, Quizi S, Youssef AM, Subhani SN, Ibrahim HM, et al. Diabetic foot complications and their risk factors from a large retrospective cohort study. PLoS One. 2015;10:e0124446.

\section{Submit your next manuscript to BioMed Central and we will help you at every step:}

- We accept pre-submission inquiries

- Our selector tool helps you to find the most relevant journal

- We provide round the clock customer support

- Convenient online submission

- Thorough peer review

- Inclusion in PubMed and all major indexing services

- Maximum visibility for your research

Submit your manuscript at www.biomedcentral.com/submit
CioMed Central 